\title{
LA DIPHTONGAISON ASCENDANTE ROMANE ET LES ÉTAPES DE L'ÉVOLUTION DU SYSTEMME VOCALIQUE DU LATIN VULGAIRE
}

\begin{abstract}
A b stract. Pawlik Janusz, La diphtongaison ascendante romane et les étapes de l'évolution du systeme vocalique du latin vulgaire [The Romance rising diphthongization and the stages of evolution of the Late Latin vowel system]. Studia Romanica Posnaniensia, Adam Mickiewicz University Press, Poznań, vol. XXIX: 2003, pp. 205-214, ISBN 83-232-1232-5, ISSN 0137-2475.

The diphthongization in the Romance languages has been a favourite subject of research for a lot of scholars over the last one hundred and fifty years. Yet, as often is the case with diachronic problems, its origin and circumstances of creation have divided the opinions of the historical grammarians. In this essay we will try to consider the diphthongization of the Vulgar Latin mid low vowels, in connection with the overall changes undergone by the Late Latin vocalic system in the stressed position.
\end{abstract}

La diphtongaison, qui est une décomposition d'une voyelle en deux phonèmes en une seule syllabe, constitue un des processus phonologiques naturels enregistrés dans de nombreuses langues du monde sans aucune parenté génétique ni structurelle. Elle appartient, à côté de la monophtongaison, aux facteurs principaux des changements dans le domaine du système vocalique. A cet égard, son action est comparable au rôle qu'a joué la palatalisation dans l'évolution des consonnes. En tant qu'exemple, on peut se servir des langues polonaise, française et anglaise. L'altérnance consonantique observée au sein du radical dans le substantif polonais ręka / ręce et dans le verbe mogę / może correspond aux modifications subies par le mot français: caelum [kelum] $>$ ciel $[$ sigl $]$ ou l'anglais: $[$ kild $]>[$ cild $]>[$ čaild $]$ child. On peut s'apercevoir de pareilles analogies dans la tendance au dédoublement des voyelles accentuées qui souvent évoluent de la même façon. L'italien a développé régulièrement la diphtongue [je] à partir de la voyelle brève latine /e/ en position libre, p. ex. PETRA > pietra, et la diphtongue [wo] à partir de la brève /o/ dans la même position: BONU > buono. Il en est de même pour le son polonais [o] qui dans les dialectes de la Silésie et de la Grande Pologne est prononcé de la même façon, soit: [wots'iets] (ojciec); [won] (on); [work'iestra] (orkiestra). Il convient de souligner que le [o] russe tonique n'est pas non plus une monophtongue (cf. очень 
[wočien']). La langue italienne, mentionné plus haut, ne représente pas cependant au sujet de la diphtongaison un schéma général caractéristique à toutes les langues romanes. Bien au contraire, quatre d'entre elles (portugais, catalan, occitan et sarde) ${ }^{1}$ n'ont pas développé de telles diphtongues à la base des voyelles moyennes du latin vulgaire, ce qui s'est produit largement en italien, espagnol, français et roumain.

On sait bien qu'une diphtongue est une jonction d'un élément vocalique, centre de la syllabe, et d'un élément supplémentaire semi-vocalique [w] ou [j]. D'une manière générale, selon que le rétrécissement des organes articulatoires suit leur ouverture ou la précède, on distingue la diphtongaison ascendante (le premier élément reste une semi-voyelle) ou la diphtongaison descendante (l'élément vocalique occupe alors la première place). Ce dernier type est particulièrement propagé dans les langues germaniques: ang. pay [pej], go [gow], mais on le retrouve également sur le territoire roman dans les langues dêjà citées, tel le portugais et le catalan: cf. port. primeiro, eu. Par contre, l'évolution du vocalisme latin sur le reste du territoire roman a pris un autre cours. Par conséquent, bien des voyelles toniques ont abouti aux diphtongues ascendantes: e $>j e ; 0>$ wo/we. De telles scissions à caractère montant sont très nombreuses en espagnol et italien modernes, et c'est à elles que nous consacrons en premier lieu cet article.

Il faut constater tout d'abord que la différence essentielle entre les diphtongues du portugais et de l'espagnol a un caractère génétique. Or, beaucoup des diphtongues descendantes du portugais actuel constituent, soit les vestiges des anciennes diphtongues latines (aurum $>$ ouro), soit se sont dégagées par suite de l'amuïssement d'une consonne intérieure (regem > rei; multum > muito). Une origine pareille reste d'ailleurs à la base de nombreuses diphtongues anglaises, p. ex. sagen > say, dag > day. En revanche, la constitution des groupes [je], [we], très répandus en espagnol, ne s'est faite qu'à partir des voyelles brèves latines [e; o].

On a beaucoup ecrit sur les circonstances et les conditions dans lesquelles eut lieu la diphtongaison romane et on est d'accord que ce fait atteignit principalement les voyelles longues. Alors, s'il en est ainsi, comment s'est-il passé que seules les voyelles brèves du latin classique ont donné naissance aux voyelles complexes, du moins en espagnol et en italien? Pour répondre à cette question, on est obligé de rappeler brièvement les étapes particulières de l'évolution des voyelles latines.

Dix sons vocaliques latins, y compris cinq longs et cinq brèves, ont perdu au fil du temps leur opposition de quantité et les voyelles nouvelles qui en surgirent ne se différaient désormais que par le degré d'aperture des organes articulatoires. Autrement dit, les anciennes voyelles longues devinrent fermées, tandis que les brèves s'ouvrirent. Il est cependant probable que les fermées $\bar{u}, \bar{o}, \bar{\jmath}, \bar{e}$ étaient toujours prononcées d'une façon un peu plus prolongée que les ouvertes

\footnotetext{
'Le provençal a généralement laissé intacts [e; o] accentués, exception faite de la séquence d'un elểment palatal qui les a parfois fait évoluer, p. ex. Pieitz<pectus; pües < posteā.
} 
correspondantes. Or, dès le $\mathrm{I}^{\mathrm{er}} \mathrm{s}$. de notre ère le vocalisme latin se présente comme il suit (Penny 1993: 40):

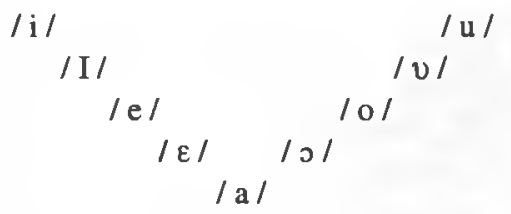

On estime que ce stade n'aurait pas pu durer longtemps et le répertoire de phonèmes vocaliques qui en résulte, en position accentuée, se réduit bientôt à sept (ibid.: 41):

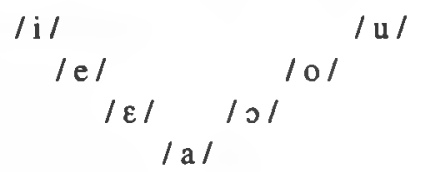

Ce changement s'est produit suivant que les phonèmes palataux $/ \mathrm{V}, \mathrm{e} / \mathrm{se}$ sont confondus et neutralisés en un seul phonème /e/, à savoir:

$\mid \mathrm{I} /, / \mathrm{e} />/ \mathrm{e} /$

et les phonèmes vélaires $/ v /, / \mathrm{o} / \mathrm{n}$ 'ont donné qu'un seul / $/$ :

$/ v /, 10 />10 /$

Cette étape du développement du latin parlé s'est maintenue à l'heure actuelle dans l'italien, portugais et catalan qui disposent précisement de sept sons vocaliques susmentionnés.

La perte des phonèmes $/ \mathrm{I}, / \mathrm{v} / \mathrm{n}$ 'a pas été la dernière phase des changements établis. Plus tard, sur un vaste territoire de l'ancien Empire Romain on assisterait à un phénomène nouveau, une fission de certaines voyelles en deux. Dans la Gaule du Nord, la diphtongaison a touché presque toutes les voyelles accentuées, dans d'autres régions, elle n'a modifié que les $/ \varepsilon /, / 0 /$ ouverts issus des $/ \mathrm{e} / \mathrm{h} / \mathrm{o}$ brefs latins. Il paraît toutefois qu'au commencement, même en Gaule, ce processus atteignit uniquement les voyelles moyennes ouvertes.

Jusqu'à présent on a émis différentes hypothèses visant à expliquer les causes de la diphtongaison romane, mais aucune n'a obtenu une approbation universelle. D'une manière générale, elles se répartissent en deux groupes fondamentaux. Le premier d'entre eux, représenté entre autres par H. Lüdtke, s'appuie sur l'inflexion en tant que force motrice des modifications vocaliques (Tekavčić 1980: 24-35). Il s'agit notamment de l'influence des /i/, / / / finals latins, c'est-à-dire des voyelles les plus fermées possible, sur les voyelles toniques qui les précèdent dans la chaîne parlée. Ces dernières se ferment lentement dans le processus d'assimilation et finissent par se diphtonguer: 
$\mid \varepsilon />/ \mathrm{e} />/ \mathrm{je} /$

$\mid 0 />/ 0 />/$ wo $/$

En conséquence, tous les sons vocaliques moyens tendent à se transformer en groupes plus complexes, y compris les anciennes voyelles fermées /o/, /e/ qui étaient au même degré susceptibles d'une telle modification. Bref, la fermeture d'une voyelle doit être considerée comme une condition indispensable et suffisante pour qu'une diphtongaison puisse démarrer. De plus, selon les partisans de cette explication la segmentation a dû atteindre aussi bien les voyelles dans les syllabes ouvertes (terminées en voyelle) que dans les fermées (terminées en consonne). Le nombre de diphtongues apparues de cette façon, serait soumis bientôt à une réduction considérable à la suite d'un processus inverse - la monophtongaison. Mais elle aura affecté seulement des groupes vocaliques dans les syllabes fermées et ceux qui sont issus des voyelles fermées /o/, /e/. Cette thèse qui consiste dans la monophtongaison sélective des diphtongues récemment constituées doit être mise en doute, car un tel processus n'aurait certainement pas épargné non plus les autres groupes vocaliques. D'autre part, cette supposition se heurte à une grave controverse en ce qui concerne l'espagnol, où la semi-voyelle [j] a plutôt freiné que favorisé la création de sons diphtongués. En voici quelques exemples opportuns:

$\begin{array}{ll}\text { FOLIA > hoja (non pas *hueja) } & \text { SUPERBIA > soberbia (non pas *sobierbia) } \\ \text { OCULU > ojo (non pas *uejo) } & \text { PREMIA > premia (non pas *priemia) } \\ \text { PODIU > poyo (non pas *pueyo) } & \text { MATERIA > madera (non pas *madiera) }\end{array}$

L'autre hypothèse, proposée jadis par les Néogrammairiens, voit les causes de la mutation dans un allongement sensible des voyelles provoqué par l'accent d'intensité (ibid.). Au centre de l'Empire (Gaule, Italie), cet allongement s'est montré beaucoup plus avancé dans une syllabe ouverte et pour cette raison la diphtongaison aura touché en principe les voyelles libres, c'est-à-dire celles qui figurent dans les syllabes ouvertes. C'est ainsi que se traduit le fait que le mot français bien $(<$ bene [be-ne]) contient la diphtongue [je], alors qu'elle manque dans le mot fer $(<$ ferrum [fer-rum]). On suppose que les sons entravés, figurant dans les syllabes fermées, ne se sont pas assez allongés pour être capables de se transmettre en diphtongues. Cette théorie a trouvé décidément plus d'adeptes dans les milieux linguistiques que la précédente (p. ex. E. Bourciez). Il est néanmoins évident qu'un allongement d'une voyelle n'entraîne pas toujours sa division imminente. Par exemple, les voyelles accentuées du portugais sont relativement plus longues que celles de l'espagnol, bien que ce soit dans ce dernier que s'est effectuée la diphtongaison ascendante ${ }^{2}$.

${ }^{2}$ Toujours est-il que des essais de la transformation directe d'une voyelle au timbre tendu en diphtongue sont observables de nos jours par quelques sujets parlant portugais (Vázquez, Mendes 1971: 75). Soit: porto [portu] $>$ [p ${ }^{w}$ ortu] vento $[$ ventu $]>\left[v^{i}\right.$ entu] . 
Si nous admettons qu'en effet l'allongement d'une voyelle aura donné lieu à sa mutation postérieure, il faut se demander si la prononciation prolongée n'a pu provoquer premièrement la fermeture du timbre vocalique. Il faut signaler à cette occasion qu'on pourrait avoir affaire à une évolution pareille à celle qui est survenue en latin classique quelques siècles auparavant, où les voyelles longues sont devenues fermées et les brèves, ouvertes. La preuve en est que les voyelles longues latines $/ \mathrm{l} /, / \overline{\mathrm{e}} /, / \overline{\mathrm{o}} /, / \overline{\mathrm{u}} /$ maintiennent jusqu'à présent le timbre fermé dans la plupart des langues romanes. Cette thèse est soutenue non seulement par $\mathrm{H}$. Luidtke ou H. Weinrich, mais aussi par R. Penny (1993: 46-47). Voilà comment il explique le phénomène de diphtongaison ayant eu lieu en Castille:

La consecuencia inmediata del alargamiento de las vocales tónicas fue el cierre de la mayoría de ellas; el incremento de la tensión muscular que requería el alargamiento debió de provocar una mayor tensión de los músculos de la boca, con la consiguiente elevación de la mandíbula y de la posición de la lengua al articular la vocal. Sin embargo, la /i/ y la /w/ no podian cerrarse más debido a su condición. Este cierre de todas las vocales, excepto las extremas, supuso una disminución en la diferencia fisiológica y acústica entre vocales vecinas; el proceso desembocó en una posible confusión. Así, por ejemplo:

/6́sso/ (< URSUS)

losso/ (< lat. vulg. OSSUM)

pudieron haberse hecho similares, llegando incluso a confundirse.

Cualquier lengua en la que esto suceda puede encontrar algún recurso terapéutico para asegurar la comprensión; el que utilizaron algunas variedades románicas, incluido el español, fue recurrir a un movimiento compensatorio de las recientemente alargadas vocales medias abiertas / $/$ / y / $/$, lo que implicaba su bimatización y la creación de incipientes diptongos:

$$
\begin{aligned}
& \mid \varepsilon />[\text { ee }]>[\text { ee }]>[\text { i } \varepsilon]>[\text { je }] \\
& / \mathrm{o} />[\text { o }]>[\text { us }]>[\text { wo }]>[\text { we }]
\end{aligned}
$$

Par ailleurs, un tel raisonnement implique une sérieuse complication. A savoir, si l'allongement d'une voyelle ouverte a abouti en effet à sa diphtongaison, pourquoi la prononciation prolongée d'une voyelle fermée n'aura pas conduit également vers sa modification? On est tenu de constater que la diphtongaison en question aurait embrassé non seulement les anciennes voyelles ouvertes, mais se serait étendue de même sur leurs variantes fermées. En réalité, ces dernières sont restées des monophtongues [e, o] sur un vaste territoire du domaine roman (spécialement en espagnol et en italien). Comment sortir donc de ce dilemme sans avoir recours à des arguments fantaisistes sur une monophtongaison ultérieure de diphtongues? Il nous paraît pourtant qu'une solution plausible s'impose assez naturellement.

De nos jours, chaque étudiant en philologie romane apprend pendant les cours de grammaire historique que les /i/ et / $\mathrm{u} /$ brefs latins ont trouvé leur continuation 
régulière dans les voyelles $/ \mathrm{e} /, \mathrm{o} / \mathrm{des}$ langues néo-latines. En témoigne le tableau qui suit:

\begin{tabular}{llll}
\multicolumn{5}{c}{ i > e } & \\
lat. & esp. & it. & port. \\
SICCUM & seco & secco & seco \\
VIRDIS & verde & verde & verde \\
PILU & pelo & pelo & pêlo \\
\multicolumn{4}{c}{ u > o } \\
CUPPA & copa & coppa & copa \\
MINUS & menos & meno & menos \\
PUTEU & pozo & pozzo & poço
\end{tabular}

Le développement susdit est confirmé aussi par des fautes d'orthographe enregistrées aux premiers siècles de $\mathrm{n}$. e. qui reflètent la vraie prononciation des voyelles précitées. Parmi les inscriptions couvrant les murs de Pompéi, on a retrouvé des formes erronées comme veces et menus au lieu de vices et minus. Enfin, un bon nombre de documents écrits, très précieux du point de vue linguistique, s'accompagne de remarques normatives des grammairiens. L'un des recueils de ce type est Appendix Probi du $\mathrm{VI}^{\mathrm{e}}$ s. qui fournit d'innombrables exemples d'erreurs manifestant des changements dans l'articulation de beaucoup de sons. En voilà quelques unes qui se rapportent à l'ouverture des $/ \mathrm{i} /, / \mathrm{u} / \mathrm{en} / \mathrm{e} / \mathrm{/} / \mathrm{o}$ (Penny 1993: 42, 52):

\section{SIRENA nON SERENA \\ TURMA nOn TORMA COLUMNA nON COLOMNA}

En même temps, il faut tenir compte des autres anomalies graphiques, très fréquentes à cette époque-là, qui témoignent aussi d'un écart existant déjà entre la langue classique et la langue parlée. Or, dès le $\mathrm{I}^{\mathrm{er}} \mathrm{s}$. de notre ère on rencontre beaucoup de méprises contraires à celles présentées auparavant. On transcrivait, par exemple (Comrie 1987: 172-173):

FILIX au lieu de FELIX

FLUS au lieu de FLOS

Il s'ensuit de cela qu'à une certaine époque l'articulation des /e/ et /o/ se sera légèrement élevée en s'approchant en termes articulatoires des $/ /$ et $/ v /$ ouverts. Cette observation trouve en partie sa confirmation dans l'ouvrage de B. Comrie (ibid.) qui la commente de la façon suivante:

/e/ and /o/ came to be raised in Latin too: hence from the first century AD onwards deviant spellings like filix, flus for felix, flos.... The raising of /e/ and /o/, which came relatively late in Latin, had occurred prehistorically in both Oscan and Umbrian. But in Oscan, whereas the resultant $/ / /$ was distinguished from existing $/ \mathrm{i} /$, as in ligatuis 'to the 
ambassadors' (<*legatois), the corresponding raised back vowel merged with $/ \mathrm{u} /$ as in fruktatius 'profit' $(<*$ fruktations).

L'auteur suggère en plus que ce traitement peut bien s'attribuer à l'influence de l'osque et de l'ombrien, deux langues où la même innovation s'était déroulée à une époque plus ancienne. En tout cas, l'abondance et la régularité de fautes similaires indiquent qu'on ne peut parler ici d'un simple concours de circonstances. Même Appendix Probi, rédigé assez tard, atteste les mêmes cas de l'écriture fautive. Si on admet que ses auteurs utilisaient une écriture strictement phonétique, on peut en conclure que dans beaucoup de mots ils articulaient effectivement / $/$ au lieu de /e/ et $/ \mathrm{w} / \mathrm{au}$ lieu de $/ \mathrm{o} /$. Voici quelques conseils du maître Probus quant à la prononciation classique des termes latins (Penny 1993: 52, 57):

\section{SENATUS non SINATUS \\ CLOACA non CLUACA}

Il ne faut pas oublier que le passage de /o/ à /u/ est un phénomène assez commun, attesté dans l'histoire de plusieurs langues. Les mots anglais good ou room, prononcés actuellement comme [gud] et [ru:m], contenaient dans le passé une voyelle longue et fermée [o:] dont l'orthographe moderne garde encore des traces. Il en est de même pour le polonais. Le graphème <ó> articulé aujourd'hui comme [u], p. ex. dans le mot góra, reflète l'état de langue moyenâgeux où ce vocable avait une articulation moins fermé [go:ra] (cf. rus. zopa). Il n'y a donc rien d'étonnant qu'une innovation semblable ait touché également le latin parlé.

Examinons les témoignages de quelques grammairiens datant des premiers siècles de notre ère dont les observations semblent confirmer la présente hypothèse. En règle générale, ils tombent d'accord que les lettres $e$ et $o$ figurant dans les mots du latin vulgaire représentent deux articulations distinctes. Les trois chercheurs d'Afrique du Nord, vivant au $V^{\mathrm{e}}$ s., affirment ce qui suit (Wright 1989: 389):

Hay cinco vocales. No todas tienen más de un sonido; dos sí, $e$ y $o$. Pues cuando la $e$ es breve, suena como un diptongo, como en equus; cuando es larga, suena como una $i$, como en demens. Igual pasa con la $o$; cuando es larga, suena dentro del paladar, como en Roma, orator; cuando es breve, se pronuncia en la parte exterior de los labios, como en opus, rosa. (Sergio)

Hay cinco vocales: $a, e, i, o, u$. Dos de éstas, $e$ y $o$, suenan de manera diferente cuando son largas que cuando son breves. Pues, cuando $o$ es larga, suena desde la parte de arriba de la boca, como en Roma; cuando es breve, suena desde los labios, como en rosa. Es lo mismo cuando la $e$ es larga, se acerca al sonido de la letra $i$, como en meta; pero cuando es breve, se aproxima al sonido de un diptongo, como en equus. (Servio)

¿Sería la $o$ larga o breve? Si es larga, el sonido saldría del paladar, como cuando se dice orator suena como se estuviera dentro, dentro del paladar. Si es breve, el sonido saldría de la parte de fuera de los labios, como de los bordes de los labios, como cuando se dice obit. Esta regla ha sido expresada por Terenciano cuando quieras pronunciarla breve, suena en la parte de fuera de los labios: cuando la pronuncies larga, suena dentro del paladar. (Pompeyo) 
L'affirmation que le $e$ long s'approche phonétiquement du son transcrit avec la lettre $i$ est d'une importance cruciale. Cela soutiendrait la thèse que le /e/ aura rétréci son timbre jusqu'à sonner plus au moins comme un $i$. Cependant, on aurait du mal à décider si cette voyelle était un $/ \mathrm{J}$, pareille à celle figurant dans le mot polonais być, ou bien un /i/ faisant partie du vocable igła. Naturellement, on peut admettre que tout en parlant d'un $e$ long il était question simplement d'une voyelle fermée $[e]$. Elle est en effet très rapprochée en termes articulatoires des unités sonores qui se transcrivent avec [i] et [I]. Au total, il serait compréhensible que les grammairiens se proposent de l'associer à la lettre $i$. Rejetons toutefois, pour l'instant, cette éventualité et essayons d'accepter que l'articulation de la voyelle en question était plus étroite au point de sonner comme une sorte de $i$.

Revenons maintenant au problème primordial de notre traité - à la diphtongaison. Nous avons constaté à plusieurs reprises que l'allongement des voyelles moyennes a anticipé leur fermeture et leur future scission en deux sons vocaliques. Néanmoins, conforme à la règle proclamant que les lois phonétiques agissent d'une manière aveugle et universelle, ce changement aurait dû atteindre aussi les voyelles /e, o/, jusqu'à alors fermées, issues des /e, o/ longs et /i, $u /$ brefs du latin classique. Or, l'acceptation de l'hypothèse que /e, o/ se proféraient comme $[\mathrm{I}, \mathrm{v}]$ (voire [ei, ow]) au moment de la diphtongaison apporte automatiquement une solution valable à ce problème. Les voyelles susdites n'auraient pas été alors capables de se diphtonguer pour la simple raison que ce processus a omis complètement les phonèmes au timbre plus fermé que $/ \mathrm{e}, \mathrm{o} /$. Donc, les voyelles ouvertes, après s'être fermées, se sont transformées en diphtongues, alors que les voyelles fermées (qui sonnaient alors comme [I, v]) ont probablement évité de partager leur sort. Certainement, cet état des choses n'a pas pu durer longtemps et finalement les sons provenant de $/ \mathrm{i}, \overline{\mathrm{e}} ; \overline{\mathrm{o}}, \mathrm{u} / \mathrm{latins}$ sont passés à leur articulation actuelle. On marquera cette étape intermédiaire (purement hypothétique) sur le schéma de l'évolution du vocalisme latin:

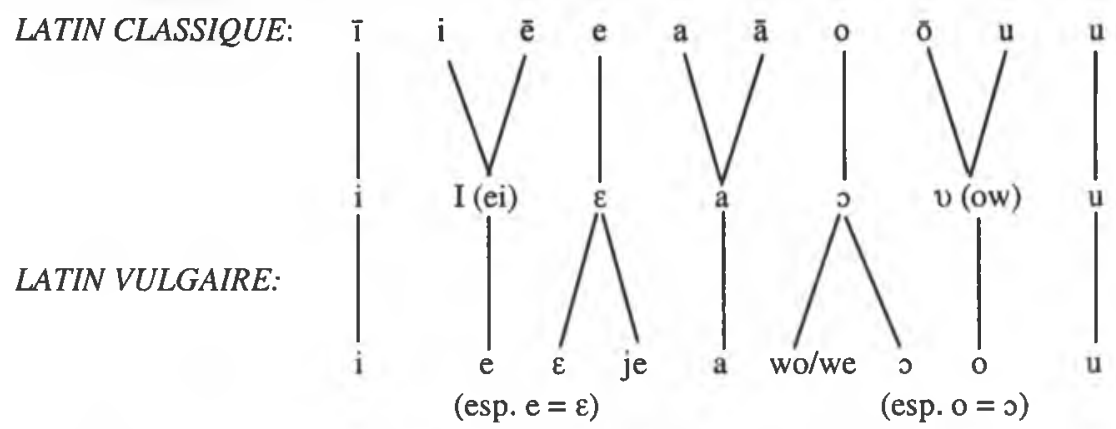

Ce diagramme donne un idée générale sur le développement supposé du vocalisme tonique dans les langues italienne et espagnole qui dorénavant ne subirait aucun changement, sauf qu'en espagnol /e, $\varepsilon /$ et /o, o/ finissent par fusionner en deux 
phonèmes nouveaux $/ \mathrm{e} / \mathrm{/} / \mathrm{o} /$. Il n'en est pas de même pour le français dont l'évolution phonétique ne s'est pas encore arrêtée. Cette langue a passé bientôt par une autre phase de diphtongaison, du type descendant, qui renfermerait à son tour les sons étroits $[\mathrm{e}, \mathrm{o}]$ (p. ex. me $>$ mei; florem $>$ flour).

Il nous reste de dire quelques mots sur le roumain, car son cas est exceptionnel. Vers la fin de l'Empire Romain, le latin d'Orient avait six voyelles distinctes, et non pas sept comme dans le reste de la Romania: $a(<a, \bar{a}), \varepsilon(<e), e(<\bar{e} i), i(<\bar{l}), o$ $(<o, \bar{o} i), u(<u, \bar{u})$. En principe, le roumain a conservé jusqu'à aujourd'hui ce vocalisme pour ses voyelles accentuées. De même qu'en espagnol, la diphtongaison roumaine se produit également devant l'entrave latine; d'où, on a non seulement ierî (< heri), mais aussi piept (< pectus). Ce qui est plus curieux encore c'est le fait qu'un autre dédoublement vocalique qui s'est opéré dans cette langue a touché aussi bien les anciens brefs comme les longs sans distinction de qualité: p. ex. $o, \bar{o}>o a$; cf. poartã (< portam), soare (< sōlem) (Bourciez 1967:549-560).

Le but essentiel du présent éxposé a été de proposer une vision différente des changements qui se sont effectués au sein du vocalisme latin tonique. Notre point de départ et de constante référence restait le phénomène de la diphtongaison ascendante des voyelles moyennes $e, o$ qui s'est réalisé au début de notre ère dans certaines langues romanes. On a admis, par ailleurs, que ce processus a affecté les unités ouvertes $[\varepsilon, \nu]$ qui, suite à leur allongement initial, vinrent à se fermer. Ce fut seulement sous cette forme qu'elles se révélèrent soumises à la fission consécutive.

Il faut pourtant remarquer que si les diphtongues [je], [wo/we] se sont créées sans le rétrécissement préalable des voyelles ci-dessus, ce qui ne peut pas s'exclure, toutes les réflexions que nous avons formulées jusqu'ici perdent leur valeur. L'articulation prolongée suivie d'une diphtongaison immédiate des phonèmes au timbre nettement différent des unités voisines fermées parvient à assûrer l'exclusivité du changement et sa limitation aux éléments, dès le début, très distincts du point de vue articulatoire. Voici comment s'est produit vraisemblablement le passage d'une voyelle isolée au complexe semi-voyelle + voyelle (cf. E. Bourciez 1967: 142-143)

$$
\begin{aligned}
& / \varepsilon />[\varepsilon:]>[\text { i } \varepsilon]>[\text { je }] \\
& / \supset />[\text { o: }]>[\text { wo }]>[\text { wo }],[\text { we }]
\end{aligned}
$$

On notera aisément qu'il y manque une phase de rétrécissement des voyelles, ce qu'il convient d'envisager comme la plus grande qualité d'une telle version d'événements. Nous évitons par là les circonstances défavorables qui nous ont empêchés d'accepter sans réserve les points de vue de certains linguistes. Il semble même que la diphtongaison en cause puisse être regardée comme une alternative à la fermeture et, peut-être, ces deux traitements ne doivent pas du tout intervenir simultanément. Une recherche expérimental en phonétique articulatoire française a démontré que lorsqu'elles s'allongent, les voyelles dites hautes $[\mathrm{i} ; \mathrm{u} ; \mathrm{e} ; \mathrm{o}]$ devien- 
nent plus étroites; tandis que les basses [ $\varepsilon$; ๖], plus relâchées, s'ouvrent encore davantage (Straka 1959: 296).

Pour terminer, nous tenons à avancer une présomption un peu audacieuse d'après laquelle la segmentation vocalique du latin parlé a pu même s'effectuer sur un élément non allongé. Cela, pour le moins, dans les zones du futur castillan et roumain où, contrairement à ce qui s'est passé en Italie et en Gaule, l'entrave n'a pas arrêté la diphtongaison. Quoi qu'il en soit, le processus dit diphtongaison romane, qui est un fait linguistique incontestable encore qu'obscur, attend toujours une explication plus cohérente et définitive.

\section{BIBLIOGRAPHIE}

B o u r c i e z, E. (1967), Elèments de linguistique romane, Klincksieck, Paris.

Co h e n, M. (1967), Histoire d'une langue: le français, Paris.

C o $\mathrm{m}$ r i e, B. (1987), The world's major languages, Croom Helm Ltd.

Encyklopedia jezzykoznawstwa ogólnego, (1993), pod red. K. Polańskiego, Ossolineum, WroclawWarszawa-Kraków.

Jezzki indoeuropejskie (1988), t. II, pod red. Leszka Bednarczuka, PWN, Warszawa.

L $l$ o y d, P.M. (1993), Del latín al español, Gredos, Madrid.

P e n n y, R. (1993), Gramática histórica del español, Ariel, Barcelona.

S t r a k a, G. (1959), Durée et timbre vocaliques. Observations de phonétique générale appliquêes à la phonétique historique des langues romanes. Zeitschrift für Phonetik und allgemeine Sprachwissenschaft, 12, 276-300.

$\mathrm{S}$ y $\mathrm{p} \mathrm{n}$ i c k i, J. (1984), Histoire externe et interne de la langue française, Wydawnictwo Naukowe UAM, Poznań.

T e k a v c i ć, P. (1980), Grammatica storica dell'italiano, Il mulino, Bologna.

Vázque z, Cuesta, P., M e ndes da L uz, M. A. (1971), Gramática da língua portuguesa. Ediçōes 70, Lisboa.

W r i g h t, R. (1989), Latín tardio y romance temprano, Gredos, Madrid. 